\title{
SOME AXIALLY SYMMETRIC ZERO MASS MESON SOLUTIONS OF EINSTEIN'S EQUATIONS
}

\author{
L. K. PATEL and V. M. TRIVEDI \\ (Received 31 July 1974; revised 30 September 1974) \\ Communicated by C. A. Hurst
}

\begin{abstract}
An axially symmetric metric in oblate spheroidal co-ordinates is considered. Two exact solutions of the field equations corresponding to zero mass meson fields are obtained. The details of the solutions are also discussed. These solutions are also generalized to include electromagnetic fields.
\end{abstract}

\section{Introduction}

Many investigaters have taken keen interest in the discussion of the coupled gravitational and zero mass meson fields in connection with investigations of the Killing horizons of static asymptotically flat fields (see Janis, Newman and Winicour (1968), Penney (1968)). The purpose of the present investigation is to discuss some axially symmetric zero mass meson fields.

The authors have been interested in certain axially symmetric static zero mass meson fields whose investigation is very much simplified by the use of oblate spheroidal co-ordinates. It is found that every solution of the field equations, corresponding to zero mass meson fields, in this coordinate system has a circular singularity which is along the focal circle besides the coordinate singularity $r=0$ on the axis.

Here it should be noted that the oblate spheroidal coordinates are used by Misra $(1960,1962)$ in connection with empty gravitational fields and electrovac universes.

\section{The co-ordinate system and the fields equations}

The oblate spheroidal co-ordinates $(\theta, \mu, \phi)$ are defined by

$$
r=a \sqrt{\left(1+\theta^{2}\right)\left(1-\mu^{2}\right)}, \quad z=a \theta \mu, \quad \phi=\phi
$$


where $r, z, \phi$ are the usual cylindrical polar co-ordinates and where $\theta$ ranges from 0 to $\infty$ and $\mu$ from -1 to 1 . The surface $\theta=0$ is a disc of radius $a$ in the $x y$ plane, with centre at the origin. The surface $\mu=+1$ is the positive $z$-axis and the surface $\mu=-1$ the negative $z$-axis. The surface $\mu=0$ is the $x y$ plane except for the part inside a circle of radius $a$ centred at the origin. The surface $\theta=$ constant $>0$ are flattened spheroids of thickness $2 a \theta$ though the axis and of radius $a \sqrt{1+\theta^{2}}$ in the equitorial plane. The surfaces $\mu=$ constant are hyperboloids of one sheet, asymptotic to the cone of semivertical angle $\cos ^{-1} \mu$ with the $z$-axis, which is the axis of the cone. We number the coordinates as $x^{1}=\theta, x^{2}=\mu, x^{3}=\phi$ and $x^{4}=t$.

The field equations corresponding to zero mass meson fields are

$$
\begin{aligned}
R_{k}^{i}-\frac{1}{2} R \delta_{k}^{i} & =-8 \pi M_{k}^{i} \\
\varphi_{.,}^{\prime} & =0
\end{aligned}
$$

where

$$
\begin{gathered}
8 \pi M_{k}^{i}=\varphi^{i} \varphi_{k}-\frac{1}{2} \delta_{k}^{i} \varphi^{i} \varphi_{l} \\
\varphi_{, k}=\varphi_{k}
\end{gathered}
$$

Here a comma and a semicolon indicate the ordinary derivative and the covariant derivative respectively. We shall take $\varphi$ to be a function of $\theta$ and $\mu$. From the above equations it is clear that

$$
R_{k}^{i}=-\varphi^{i} \varphi_{k} \text {. }
$$

We have taken $\varphi$ as a function of $\theta$ and $\mu$. Thus $R_{3}^{3}=R_{4}^{4}=0$.

Now, the static axially symmetric line-element with $R_{3}^{3}+R_{4}^{4}=0$ can be expressed as

$$
d s^{2}=-e^{H}\left(d r^{2}+d z^{2}\right)-r^{2} e^{-\kappa} d \phi^{2}+e^{\kappa} d t^{2}
$$

where $H=H(r, z)$ and $K=K(r, z)$ (see Synge (1960)). Transforming (6) to the coordinates $(\theta, \mu, \phi)$ defined by $\left({ }^{*}\right)$ we obtain

$$
\begin{aligned}
d s^{2}= & -a^{2}\left(\theta^{2}+\mu^{2}\right) e^{H}\left(\frac{d \theta^{2}}{1+\theta^{2}}+\frac{d \mu^{2}}{1-\mu^{2}}\right) \\
& -a^{2}\left(1+\theta^{2}\right)\left(1-\mu^{2}\right) e^{-\kappa} d \phi^{2}+e^{\kappa} d t^{2}
\end{aligned}
$$

where $H$ and $K$ are both functions $\theta$ and $\mu$. Here it should be noted that the Jacobian of transformation $\left(^{*}\right)$ is given by

$$
J=a^{2}\left(\theta^{2}+\mu^{2}\right)\left\{\left(1+\theta^{2}\right)\left(1-\mu^{2}\right)\right\}^{-\frac{1}{2}}
$$

It is clear that $J$ vanishes on the focal circle $(\theta=0, \mu=0)$. Accordingly the inverse transformation will not exist here. 
The non-zero components of the Ricci tensor $\boldsymbol{R}_{k}^{i}$ corresponding to (7) are

$$
\begin{aligned}
& R_{1}^{\prime}=\frac{e^{-H}}{2 a^{2}\left(\theta^{2}+\mu^{2}\right)}\left[-\left(1+\theta^{2}\right)\left(H_{11}+K_{1}^{2}\right)-\left(1-\mu^{2}\right) H_{22}+2 \theta K_{1}+2 \mu H_{2}\right], \\
& R_{2}^{2}=\frac{e^{-H}}{2 a^{2}\left(\theta^{2}+\mu^{2}\right)}\left[-\left(1+\theta^{2}\right) H_{11}-\left(1-\mu^{2}\right)\left(H_{22}+K_{2}^{2}\right)-2 \theta H_{1}-2 \mu K_{2}\right],
\end{aligned}
$$

$$
\begin{gathered}
R_{3}^{3}=-R_{4}^{4}=\frac{e^{-H}}{2 a^{2}\left(\theta^{2}+\mu^{2}\right)}\left[\left(1+\theta^{2}\right) K_{11}+2 \theta K_{1}+\left(1-\mu^{2}\right) K_{22}-2 \mu K_{2}\right], \\
\frac{R_{1}^{2}}{1-\mu^{2}}=\frac{R_{2}^{1}}{1+\theta^{2}}=\frac{e^{-H}}{2 a^{2}\left(\theta^{2}+\mu^{2}\right)}\left[-K_{1} K_{2}-\frac{\mu\left(H_{1}+K_{1}\right)}{1-\mu^{2}}+\frac{\theta\left(H_{2}+K_{2}\right)}{1+\theta^{2}}\right]
\end{gathered}
$$

Here and in what follows lower suffixes 1 and 2 after an unknown function imply a partial differentiation with respect to $\theta$ and $\mu$ respectively.

The field equations (1) and (2) together with the results (8) give us the following five equations to determine the three unknown functions $K H$ and $\varphi$

$$
\begin{aligned}
& H_{1}+K_{1}=\frac{\theta\left(1+\theta^{2}\right)\left(1-\mu^{2}\right)}{\theta^{2}+\mu^{2}}\left(\varphi_{1}^{2}+\frac{1}{2} K_{1}^{2}\right)-\frac{\theta\left(1-\mu^{2}\right)^{2}}{\theta^{2}+\mu^{2}}\left(\varphi_{2}^{2}+\frac{1}{2} K_{2}^{2}\right) \\
& -\frac{\mu\left(1+\theta^{2}\right)\left(1-\mu^{2}\right)}{\theta^{2}+\mu^{2}}\left(K_{1} K_{2}+2 \varphi_{1} \varphi_{2}\right) \\
& H_{2}+K_{2}=\frac{\mu\left(1+\theta^{2}\right)^{2}}{\theta^{2}+\mu^{2}}\left(\varphi_{1}^{2}+\frac{1}{2} K_{1}^{2}\right)-\frac{\mu\left(1-\mu^{2}\right)\left(1+\theta^{2}\right)}{\theta^{2}+\mu^{2}}\left(\varphi_{2}^{2}+\frac{1}{2} K_{2}^{2}\right) \\
& +\frac{\theta\left(1+\theta^{2}\right)\left(1-\mu^{2}\right)}{\theta^{2}+\mu^{2}}\left(K_{1} K_{2}+2 \varphi_{1} \varphi_{2}\right) \\
& \left(1+\theta^{2}\right) K_{11}+\left(1-\mu^{2}\right) K_{22}+2 \theta K_{1}-2 \mu K_{2}=0 \\
& \left(1+\theta^{2}\right) \varphi_{11}+\left(1-\mu^{2}\right) \varphi_{22}+2 \theta \varphi_{1}-2 \mu \varphi_{2}=0 \\
& \left(1+\theta^{2}\right) H_{11}+\left(1-\mu^{2}\right) H_{22}+\frac{1}{2}\left(1+\theta^{2}\right) K_{1}^{2}+\frac{1}{2}\left(1-\mu^{2}\right) K_{2}^{2} \\
& +\theta H_{1}-\theta K_{1}+\mu K_{2}-\mu H_{2}=-\left(1+\theta^{2}\right) \varphi_{1}^{2}-\left(1-\mu^{2}\right) \varphi_{2}^{2}
\end{aligned}
$$

It can be verified that the equation (13) follows from the equations (9), (10), (11) and (12). Therefore (9), (10), (11) and (12) are the four independent equations to determine $K H$ and $\varphi$.

The last two equations (11) and (12) are Laplace's equations in oblate spherioidal coordinates. It is well known that the Laplace's equation in oblate spheroidal coordinates has solutions of the form $A_{n} P_{n}(\mu) q_{n}(\theta)$ where $A_{n}$ are 
constants and $p_{n}(\mu), q_{n}(\theta)$ are Legendre functions of order $n$. It has been pointed out by Hobson (1931) that at a point on the focal circle $\theta=0, \mu=0$, the function $p_{n}(\mu) q_{n}(\theta)$ has an infinite differential coefficient in the direction normal to one of the hypersurfaces $\theta=$ constant or $\mu=$ constant. Hence every solution represented by $p_{n}(\mu) q_{n}(\theta)$ will be singular on the focal circle.

\section{The solutions of the field equations for zero mass meson fields}

In this paper we shall limit ourselves to the cases $n=0$ and $n=1$ for $p_{n}(\mu) q_{n}(\theta)$. We know that $p_{0}(\mu)=1 . q_{0}(\theta)=\cot ^{-1} \theta, p_{1}(\mu)=\mu$ and $q_{1}(\theta)=$ $1-\theta \cot ^{-1} \theta$. We consider the following two cases

$$
\begin{aligned}
& K=A \cot ^{-1} \theta, \quad \varphi=B \cot ^{-1} \theta \\
& K=A \mu\left(1-\theta \cot ^{-1} \theta\right), \quad \varphi=B \mu\left(1-\theta \cot ^{-1} \theta\right)
\end{aligned}
$$

Here $A$ and $B$ are constants.

In these two cases the expressions for the function $H$ are given by

$$
\text { (i) } \begin{aligned}
H= & -A \cot ^{-1} \theta+\frac{1}{2}\left(B^{2}+\frac{1}{2} A^{2}\right) \log \frac{\theta^{2}+\mu^{2}}{\theta^{2}+1}+\alpha \\
H= & -A \mu\left(1-\theta \cot ^{-1} \theta\right) \\
& +\left(B^{2}+\frac{A^{2}}{2}\right)\left[\theta \cot ^{-1} \theta-\frac{1}{2}\left(1+\theta^{2}\right)\left(\cot ^{-1} \theta\right)^{2}+\frac{1}{2} \log \frac{1+\theta^{2}}{\theta^{2}+\mu^{2}}\right] \\
& +\left(B^{2}+\frac{A^{2}}{2}\right) \frac{\mu^{2}}{2}\left\{1-\theta \cot ^{-1} \theta+\left(1+\theta^{2}\right)\left(\cot ^{-1} \theta\right)^{2}\right\}+\beta
\end{aligned}
$$

where $\alpha$ and $\beta$ are integration constants. Thus (7), (14), and (16) constitute the first solution and (7), (15) and (17) constitute the second solution of the field equations for zero mass meson fields.

These two solutions have a singularity on the focal circle (that is, $\theta=0$ and $\mu=0$ ).

It can be easily verified that when $B=0$ the zero mass meson field disappears and the solutions discussed above reduce to the vacuum solutions $\left(R_{k}^{i}=0\right)$ discussed by Misra (1960).

\section{Solutions of the coupled Einstein-Maxwell scalar field equations}

The Einstein-Maxwell scalar field equations are

$$
R_{k}^{i}-\frac{1}{2} \delta_{k}^{i} R=-8 \pi T_{k}^{\prime}, \quad F_{, k}^{k}=0, \quad F_{[i k: l]}=0, \quad \varphi_{, i}^{\prime}=0,
$$

with $T_{k}^{i}=E_{k}^{i}+M_{k}^{i}$ where

$$
4 \pi E_{k}^{i}=F^{i l} F_{k l}-\frac{1}{4} \delta_{k}^{i} F_{l m} F^{l m}, \quad 8 \pi M_{k}^{i}=\varphi^{i} \varphi_{k}-\frac{1}{2} \delta_{k}^{i} \varphi^{\prime} \varphi_{1}
$$


Pandey (1970) has described a method to generate a solution of coupled Einstein-Maxwell scalar field equations from a known solution of the scalar (zero mass meson) field equations. For this purpose we consider three metrics.

$$
\begin{aligned}
& d s^{2}=e^{2 u} d t^{2}+e^{-2 u} d s^{* 2} \\
& d \tilde{s}^{2}=e^{2 w} d t^{2}+e^{-2 w} d s^{* 2} \\
& d s^{* 2}=h_{\alpha \beta} d x^{\alpha} d x^{\beta} \quad(\alpha, \beta=1,2,3)
\end{aligned}
$$

where $u, w$ and $h_{\alpha \beta}$ are functions of $x^{1}, x^{2}, x^{3}$. Pandey (1970) has shown that if (19) describes a solution of the field equations with the mass-less scalar meson fields as source, (20) gives a solution of the coupled Einstein-Maxwell equations with non-vanishing electromagnetic field where $e^{w}=\lambda$ sech $u$ and $F_{v k}$ are given by

$$
F^{\alpha \beta}=(-g)^{-\frac{1}{2}} \epsilon^{\alpha \beta \gamma} \cos N C_{, \gamma}, \quad F_{4 \alpha}=\sin N C_{, \alpha} .
$$

Here $C=\lambda \tanh u, \lambda$ and $N$ are constants and $g$ is the determinant of the metric tensor of the line-element (20).

The line-element (7) can be expressed as

$$
\begin{aligned}
d s^{2}=e^{\kappa} d t^{2}-e^{-\kappa} & {\left[a^{2}\left(\theta^{2}+\mu^{2}\right) e^{s}\left(\frac{d \theta^{2}}{1+\theta^{2}}+\frac{d \mu^{2}}{1-\mu^{2}}\right)\right.} \\
& \left.+a^{2}\left(1+\theta^{2}\right)\left(1-\mu^{2}\right) d \phi^{2}\right]
\end{aligned}
$$

where $S=H+K$. Thus the metric (23) is of the form (19). In section 3 we have derived two solutions for massless scalar meson fields. Applying the above mentioned method we can obtain two exact solutions of the coupled EinsteinMaxwell equations, (18), described by the metric (20). These two solutions are listed below.

Solution (i):

$$
\begin{aligned}
e^{\omega} & =\lambda \operatorname{sech}(K / 2), \quad K=A \cot ^{-1} \theta, \quad C=\lambda \tanh (K / 2), \\
S & =\frac{1}{2}\left(B^{2}+\frac{1}{2} A^{2}\right) \log \frac{\theta^{2}+\mu^{2}}{\theta^{2}+1}+\alpha
\end{aligned}
$$

Solution (ii):

$$
\begin{aligned}
e^{w}= & \lambda \operatorname{sech}(K / 2), \quad K=A \mu\left(1-\theta \cot ^{-1} \theta\right), \quad C=\lambda \tanh (K / 2), \\
S= & \left(B^{2}+\frac{A^{2}}{2}\right)\left[\theta \cot ^{-1} \theta-\frac{1}{2}\left(1+\theta^{2}\right)\left(\cot ^{-1} \theta\right)^{2}+\log \frac{1+\theta^{2}}{\theta^{2}+\mu^{2}}\right] \\
& +\frac{\mu^{2}}{2}\left(B^{2}+\frac{A^{2}}{2}\right)\left[1-2 \theta \cot ^{-1} \theta+\left(1+\theta^{2}\right)\left(\cot ^{-1} \theta\right)^{2}\right]+\beta
\end{aligned}
$$


The explicit expressions for the electromagnetic field tensor $F_{i k}$ for the above solutions can be obtained with the help of the result (22). From (22) it is clear that when $N=0$ we get purely magnetic fields and when $N=\pi / 2$ we get purely electric fields. The above two solutions have singularities on the focal circle.

The authors would like to thank Dr. M. Misra for many valuable discussions. The authors are grateful to the referee for many helpful comments.

\section{References}

E. W. Hobson (1931), The theory of spherical and ellipsoidal harmonics (Cambridge University Press, 1931).

A. I. Janis, E. T. Newman and J. Winicour (1968), 'Reality of the Schwarzschild singularity', Phys. Rev. Lett. 20. 878-880.

M. Misra (1960), 'Some axially symmetric empty gravitational fields', Proc. Nat. Acad. Sci. India Sect. A 26, 673-680.

M. Misra (1962), 'Electrovac universes', Proc. Nat. Acad. Sci. India Sect. A 28, 105-119.

D. B. Pandey (1970), Contributions to the problems of axisymmetric fields in general relativity (Ph. D. Thesis, University of Gorakhpur, Gorakhpur, India).

R. Penney (1968), 'Axially symmetric zero mass meson solutions of Einstein's equations', Phys. Rev. 174, 1578-1579.

J. L. Synge (1960), Relativity - The General Theory (North Holland Publishing Co., Amsterdam, $1960)$.

Departments of Mathematics and Physics, Gujarat University, Ahmedabad 380009, India. 